TRANSACTIONS OF THE

AMERICAN MATHEMATICAL SOCIETY

Volume 354, Number 5, Pages 1899-1908

S 0002-9947(01)02933-6

Article electronically published on November 21, 2001

\title{
PRINCIPAL BUNDLES OVER A PROJECTIVE SCHEME
}

\author{
DONGHOON HYEON
}

\begin{abstract}
We prove the existence of a quasi-projective moduli scheme for principal bundles over an arbitrary projective scheme.
\end{abstract}

Moduli of principal bundles over a nonsingular complex projective curve was constructed by A. Ramanathan in his thesis, written in 1976 and published recently in 13 . In this paper, we generalize the method and construct a moduli scheme of stable principal bundles over a projective scheme $X$. Moduli space of principal bundles over a nonsingular projective curve has attracted much attention in the mid 90 s in view of the celebrated Verlinde formula. Also, Friedman, Morgan and Witten studied them with $F$-theory from physics in mind ([5], [6], [7]), and R.Donagi wrote a series of papers on principal bundles over an elliptic fibration in a similar vein, applying the theory to the heterotic string and $F$-theory duality ([1] with $\mathrm{P}$. Aspinwall, 3], and [4]). The introduction of [5] contains a more comprehensive history of the subject.

In Section 1, we give basic definitions and lemmas. In Section 2, we will briefly review the construction of the moduli scheme of vector bundles, and prove the crucial result on the linearization of an algebraic group action on a Grothendieck's quot scheme over a general base scheme. In Section 3, we show the existence of the coarse moduli scheme of stable $k^{*} \times \operatorname{Aut}(\mathfrak{g})$-bundles, and in Section 4 we will explain how this leads to the construction of moduli of $G$-bundles for general reductive $G$.

Although we succeed in obtaining a quasi-projective moduli scheme, we do not know how to compactify it because of the lack of any notion corresponding to the torsion-freeness of sheaves. One knows from the vector bundle case that adding semistable bundles is not enough to achieve a compactification. However, it is certainly worthwhile to construct a moduli scheme of semistable principal bundles, and the author plans to do further research in that direction. Another thing that leaves room for improvement is our definition of stability, which is not intrinsic and depends on the chosen representation. (See Theorem 2.2 of [5] for various stability conditions equivalent to the intrinsic stability of Ramanathan.) In the last section, we will compare our notion of stability and that of Ramanathan, and note that by adding polystable bundles, our moduli would contain all the bundles polystable in the sense of Ramanathan.

\section{Notations}

Unless otherwise stated,

1. $k$ is an algebraically closed field of characteristic 0 .

Received by the editors September 4, 2000 and, in revised form, March 8, 2001.

2000 Mathematics Subject Classification. Primary 14D20.

The author thanks Chris Sim at 3Com for his computing equipment support.

(C)2001 American Mathematical Society 
2. A scheme will always mean an algebraic scheme, i.e., a scheme of finite type over $k$.

3. $X$ will be a projective scheme over $k$ with a chosen polarization $\mathcal{O}_{X}(1)$.

4. $G$ is a connected reductive algebraic group over $k$, and $\mathfrak{g}$ will denote the Lie algebra of $G$.

5. $P$ is a polynomial with rational coefficients.

6. $(\mathbf{S c h} / k)$ will denote the category of schemes of finite fype over $k$ with the faithfully flat topology on it.

\section{Preliminaries}

Definition 1. Let $X$ be a scheme. A principal $G$-bundle (or just a $G$-bundle for convenience) over $X$ is an $X$-scheme $p: E \rightarrow X$ on which $G$ acts from the right such that

1. $p$ is faithfully flat and $G$-invariant,

2. $p_{1} \times \sigma: E \times G \longrightarrow E \times{ }_{X} E$ defined by $(x, g) \mapsto(x, \sigma(x, g))$ is an isomorphism, where $\sigma: E \times G \rightarrow E$ denotes the group action. One may replace the second condition by the condition that $p$ is locally trivial in the faithfully flat topology, i.e., for any open subscheme $U \stackrel{i}{\hookrightarrow} X$ there exists a faithfully flat $U$-scheme $f: U^{\prime} \rightarrow U$ such that $f^{*} i^{*} E \simeq U^{\prime} \times G$.

Now we define the stability condition for principal bundles. The well-known stability condition for vector bundles is an essential part of it. (To recall it, see the beginning of section 2]

Definition 2. A principal $G$-bundle $E$ over $X$ is said to be stable (resp. semistable, polystable) with Hilbert polynomial $P$ if its adjoint bundle $E \times^{G} \mathfrak{g}$ is stable (resp. semistable, polystable) with Hilbert polynomial $P$.

Remark 1. We will often drop the words 'with Hilbert polynomial $P$ ' when there is no risk of confusion.

We are interested in the moduli functor $\underline{\mathbf{M}}_{G, P}:(\mathbf{S c h} / k) \rightarrow($ Sets $)$ defined by

\section{Definition 3.}

$$
\underline{\mathbf{M}}_{G, P}(S)=\left\{E \rightarrow S \times X \mid \begin{array}{ll}
E \text { is a } G \text {-bundle such that } \\
\left.E\right|_{s \times X} \text { is stable for all } s \in S
\end{array}\right\} / \sim .
$$

Here $\sim$ means just the equivalence relation given by isomorphisms.

To construct a coarse moduli scheme for $\underline{\mathbf{M}}_{G, P}$, we shall follow the strategy of 13. In short, it is to use the adjoint representation $\mathrm{Ad}: G \rightarrow \mathrm{GL}(\mathfrak{g})$ and construct a moduli space of $G$-bundles from the known moduli space of $\operatorname{GL}(\mathfrak{g})$-bundles.

Definition 4. Let $\mathcal{S}$ be a set of isomorphism classes of $G$-bundles on $X$, and $F_{\mathcal{S}}$ a functor from $(\mathbf{S c h} / k)$ to (Sets) defined by

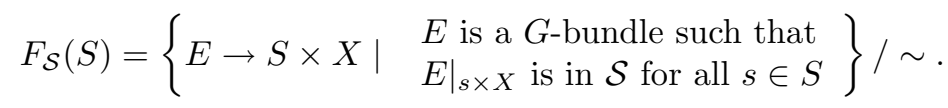

Again, $\sim$ denotes the equivalence relation given by isomorphisms. Suppose that $M$ is a scheme on which an algebraic group $H$ acts by $\alpha: M \times H \rightarrow M$. $M$ is said to be an $H$-universal space for $F_{\mathcal{S}}$ (or for $\mathcal{S}$ ) if there is an isomorphism of sheaves between $\tilde{F}_{\mathcal{S}}$ and $(M / H)^{\sim}$, where $M / H$ is the functor from $(\mathbf{S c h} / k)$ to (Sets) defined by

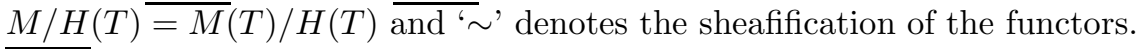


Our first lemma follows easily from the definition of the universal space (see Proposition 4.5 [13]).

Lemma 1. Suppose that $M$ is an $H$-universal space for $\underline{\mathbf{M}}_{G, P}$ and that it has a good quotient $\phi: M \rightarrow M / / H$. Then $M / / H$ is a coarse moduli scheme for $\underline{\mathbf{M}}_{G, P}$.

Definition 5. Let $F_{\mathcal{S}}$ be as above, and let $\mathcal{E} \rightarrow \mathcal{R} \times X$ be a family of $G$-bundles in $\mathcal{S}$. Suppose that $H$ acts on $\mathcal{R}$ by $\alpha: \mathcal{R} \times H \rightarrow \mathcal{R}$, and also on $\mathcal{E}$ compatibly. Then $\mathcal{E} \rightarrow \mathcal{R} \times X$ is called an $H$-universal family for $F_{\mathcal{S}}$ (or for $\mathcal{S}$ ) if it has the following properties.

1. For any family of $G$-bundles $\mathcal{F} \rightarrow S \times X$ in $\mathcal{S}$ and any $s \in S$, there exist an open neighborhood $U$ of $s$ and a morphism $f_{s}: U \rightarrow \mathcal{R}$ such that $\mathcal{F}_{U} \simeq \mathcal{E}_{f_{s}}=$ $\left(f_{s} \times 1_{X}\right)^{*} \mathcal{E}$.

2. For any two morphisms $f_{1}, f_{2}: S \rightarrow \mathcal{R}$ and an isomorphism $f_{12}: \mathcal{E}_{f_{1}} \stackrel{\sim}{\rightarrow} \mathcal{E}_{f_{2}}$, there exists a unique morphism $h: S \rightarrow H$ such that $f_{2}=\alpha \circ\left(f_{1} \times h\right)$ and $f_{12}=\left(f_{1} \times h \times 1_{X}\right)^{*}(\tilde{\alpha})$, where $\tilde{\alpha}$ is the isomorphism from $\mathcal{E} \times H$ to $\left(\alpha \times 1_{X}\right)^{*} \mathcal{E}$ given by the $H$-action on $\mathcal{E}$.

Remark 2. If $\mathcal{E} \rightarrow \mathcal{R} \times X$ is an $H$-universal family, then $\mathcal{R}$ is an $H$-universal space.

Lemma 2. Let $\rho: K \rightarrow G$ be a morphism of algebraic groups and $\mathcal{E} \rightarrow \mathcal{R} \times X$ a family of $G$-bundles. Let $\Gamma(\rho, \mathcal{E})$ be the functor $(\mathbf{S c h} / \mathcal{R}) \rightarrow($ Sets $)$ defined by

$$
\Gamma(\rho, \mathcal{E})(S)=\left\{(E, \phi) \mid \begin{array}{l}
E \text { is a K-bundle over } S \times X, \\
\phi \text { is an isomorphism } \rho_{*}(E) \stackrel{\sim}{\rightarrow} \mathcal{E}_{S}
\end{array}\right\} / \sim
$$

where $\sim$ denotes the equivalence relation given by isomorphisms and $\rho_{*}(E)$ is the associated $G$-bundle $E \times{ }^{K} G=(E \times G) / K$. If $\rho$ is immersive, then $\Gamma(\rho, \mathcal{E})$ is representable by a quasi-projective $\mathcal{R}$-scheme.

In fact, when $\rho$ is immersive, $\Gamma(\rho, \mathcal{E})$ is isomorphic to the functor of sections $\operatorname{Hom}_{\mathcal{R} \times X}(\mathcal{R} \times X, \mathcal{E} / K)$ which is representable by an open subscheme of the quasiprojective $\mathcal{R}$-scheme $\mathbf{H i l b}_{\mathcal{R}}(\mathcal{E} / K)[9$. Another key lemma is

Lemma 3 (Lemma 4.10, [13]). Let $G, K$ be algebraic groups and $\rho: K \longrightarrow G$ a group homomorphism. Suppose that $\mathcal{E} \rightarrow \mathcal{R} \times X$ is an $H$-universal family for a set $\mathcal{S}$ of $G$-bundles. Suppose further that $\Gamma(\rho, \mathcal{E})^{\sim}$ is representable by a scheme $\mathcal{R}_{1}$. Then:

1. $H$ acts on $\mathcal{R}_{1}$ in a natural way, and $\mathcal{R}_{1}$ with this action is an $H$-universal family for the set $\mathcal{S}^{\prime}$ of $K$-bundles which give $G$-bundles in $\mathcal{S}$ on extending the structure group by $\rho: K \longrightarrow G$.

2. If $\rho$ is injective so that $\Gamma(\rho, \mathcal{E})$ itself is representable, then the universal element $\mathcal{E}_{1} \longrightarrow \mathcal{R}_{1} \times X$ in $\Gamma(\rho, \mathcal{E})\left(\mathcal{R}_{1}\right)$ is an $H$-universal family for $\mathcal{S}^{\prime}$.

Due to this lemma, via the immersive adjoint representation Ad $: G / Z(G) \rightarrow$ $\operatorname{GL}(\mathfrak{g})$ we can deduce a universal family of $G / Z(G)$-bundles from a universal family of $\mathrm{GL}(\mathfrak{g})$-bundles. This is a cornerstone of our construction.

We will review the construction of a universal family of $\mathrm{GL}(\mathfrak{g})$-bundles in the next section.

\section{Hilbert SCHEMEs AND MOdUli OF VeCtor BUNDLES}

In this section and later on, we will denote a $\mathrm{GL}_{n}$-bundle and its associated vector bundle of rank $n$ by the same letter. Also, we will not distinguish between 
a vector bundle and its locally free sheaf of sections. A vector bundle $E$ on $X$ is said to be semistable (resp. stable) if $E$ is pure and for every proper subsheaf $F$ of $E, \frac{P_{E}}{\operatorname{rank}(E)} \geq(>) \frac{P_{F}}{\operatorname{rank}(F)}$ holds, where $P_{E}$ and $P_{F}$ are the Hilbert polynomials and ' $\geq$ ' (and ' $>$ ') is defined by the lexicographic order on $\mathbb{Q}[T]$.

Let $\mathcal{S}_{P}$ denote the collection of all stable vector bundles on $X$ with Hilbert polynomial $P$. There exists an integer $m$ such that for any $E \in \mathcal{S}_{P}, E(m)$ is generated by sections and $H^{i}(X, E(m))=0$ for all $i>0$. Then $h^{0}(X, E(m))$ is fixed for all $E \in \mathcal{S}_{P}$, which we call $N$. Let $V=k^{N}$. It follows that $\mathbf{Q u o t}_{V \otimes \mathcal{O}_{X}(-m) / X / k}^{P}$ parametrizes all the bundles in $\mathcal{S}_{P}$. Let $\mathcal{R}^{\prime}$ be the locally closed subscheme of Quot $_{V \otimes \mathcal{O}_{X}(-m) / X / k}^{P}$ consisting of the points $\left[V \otimes \mathcal{O}_{X}(-m) \rightarrow E\right]$ where $E$ is locally free and the induced map of the cohomology $V \rightarrow H^{0}(X, E(m))$ is an isomorphism. It was shown by C. Simpson [16, by using Grothendieck's embedding of the quot scheme in a Grassmannian variety, that one can linearize the $\operatorname{GL}(V)$ action on $\mathcal{R}^{\prime}$, such that $\mathcal{R}^{\prime} s / / \mathrm{GL}(V)$ is the moduli scheme of stable vector bundles with Hilbert polynomial $P$. (It is written up in full generality in 11].) Let $\mathcal{R}=\mathcal{R}^{\prime} s$. From the universal quotient sheaf over $\mathbf{Q u o t}_{V \otimes \mathcal{O}_{X}(-m) / X / k}^{P} \times X$, one can easily produce a $\mathrm{GL}(V)$-universal family $\mathcal{E} \rightarrow \mathcal{R} \times X$ of stable vector bundles with Hilbert polynomial $P$.

Now we study an algebraic group action on a quot scheme over a general base scheme, which we will use in the next section.

Proposition 1. Let $S$ be a scheme of finite type over $k$, and let $f: Z \rightarrow S$ be a projective morphism of schemes. Let $\Gamma$ be an algebraic group over $k$ acting on $Z$ and $S$ linearly with respect to ample line bundles $L_{1}$ over $Z$ and $L_{2}$ over $S$, respectively, and suppose that $f$ is $\Gamma$-equivariant. Then for any $\Gamma$-linearized $S$-flat coherent sheaf $\mathcal{F}$ over $Z$ and a Hilbert polynomial $P_{0}$, we have the following.

1. $\Gamma$ acts on $\mathbf{Q u o t}_{\mathcal{F} / Z / S}^{P_{0}}$, and the canonical morphism $p: \operatorname{Quot}_{\mathcal{F} / Z / S}^{P_{0}} \rightarrow S$ is $\Gamma$-equivariant.

2. There exists a $\Gamma$-linearized line bundle on $\mathbf{Q u o t}_{\mathcal{F} / Z / S}^{P_{0}}$ which is very ample relative to $p$.

Proof. 1. The action of $\Gamma$ on $\operatorname{Quot}_{\mathcal{F} / Z / S}^{P_{0}}:$ Let $\sigma: S \times \Gamma \rightarrow S$ be the $\Gamma$-action on $S$, and $\Sigma: Z \times \Gamma \rightarrow Z$, that on $Z$. Since $f$ is $\Gamma$-equivariant, we have a unique isomorphism $\gamma: Z \times \Gamma \stackrel{\sim}{\rightarrow} \sigma^{*} Z$ such that the diagram

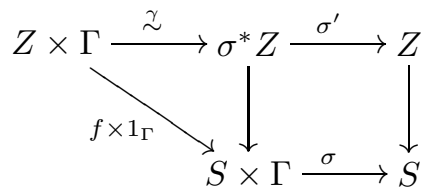

commutes and $\Sigma=\sigma^{\prime} \circ \gamma$, where $\sigma^{\prime}$ is the base change of $\sigma$.

Let $t: T \rightarrow S$ be a $T$-point of $S$ and $g: T \rightarrow \Gamma$ a $T$-point of $\Gamma$. Also, let $t \cdot g$ denote the $T$-point of $S$ defined by $T \stackrel{t \times g}{\rightarrow} S \times \Gamma \stackrel{\sigma}{\rightarrow} S$. It is just $\sigma_{T}(t, g)$, where $\sigma_{T}$ is the $\Gamma(T)$ action on $S(T)$ coming from $\sigma$. Consider the two $T$-schemes $Z_{t}=t^{*} Z$ and $Z_{t \cdot g}=(t \cdot g)^{*} Z$ gotten by the base change. Since $Z_{t}=(t \times g)^{*}(Z \times \Gamma)$ and $Z_{t \cdot g}=(t \times g)^{*}\left(\sigma^{*} Z\right)$, we have the isomorphism $g^{\prime}:=(t \times g)^{*} \gamma: Z_{t} \stackrel{\sim}{\rightarrow} Z_{t \cdot g}$.

Now let $\mathcal{F}_{t}$ and $\mathcal{F}_{t \cdot g}$ denote $t^{\prime} \mathcal{F}$ and $(t \cdot g)^{\prime}{ }^{*} \mathcal{F}$, respectively, where $t^{\prime}: Z_{t} \rightarrow Z$ and $(t \cdot g)^{\prime}: Z_{t \cdot g} \rightarrow Z$ are the base changes of $t$ and $t \cdot g$. Then we have an isomorphism of the sheaves $\mathcal{F}_{t}$ and $g^{*} \mathcal{F}_{t \cdot g}$ coming from the linearization of $\mathcal{F}$. In fact, let 
$\Phi: \Sigma^{*} \mathcal{F} \stackrel{\sim}{\rightarrow} p_{Z}^{*} \mathcal{F}$ be the linearization, and consider the following commutative diagram:

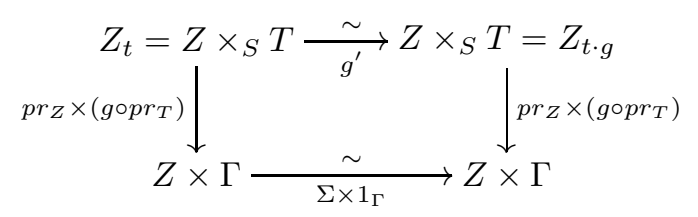

where $p r_{Z}$ and $p r_{T}$ are projections from $Z \times_{S} T$ to $Z$ and $T$, respectively. Then we have $\mathcal{F}_{t}=\left(p r_{Z} \times\left(g \circ p r_{T}\right)\right)^{*} p_{Z}^{*} \mathcal{F}$. On the other hand, we also have

$$
\begin{aligned}
g^{\prime} \mathcal{F}_{t \cdot g} & =g^{\prime}\left(p r_{Z} \times\left(g \circ p r_{T}\right)\right)^{*} p_{Z}^{*} \mathcal{F}=\left(p r_{Z} \times\left(g \circ p r_{T}\right)\right)^{*}\left(\Sigma \times 1_{\Gamma}\right)^{*} p_{Z}^{*} \mathcal{F} \\
& =\left(p r_{Z} \times\left(g \circ p r_{T}\right)\right)^{*} \Sigma^{*} \mathcal{F} .
\end{aligned}
$$

Hence we have the isomorphism $\left(p r_{Z} \times\left(g \circ p r_{T}\right)\right)^{*} \Phi: g^{\prime} * \mathcal{F}_{t \cdot g} \stackrel{\sim}{\rightarrow} \mathcal{F}_{t}$.

Then $\Gamma$ acts on $\mathbf{Q u o t}_{\mathcal{F} / Z / S}^{P_{0}}$ by

$$
\left[\mathcal{F}_{t} \stackrel{q}{\rightarrow} E\right] \cdot g=\left[\mathcal{F}_{t \cdot g} \stackrel{\left(p r_{Z} \times\left(g \circ p r_{T}\right)\right)^{*} \Phi^{-1}}{\longrightarrow} g^{\prime-1 *} \mathcal{F}_{t} \stackrel{g^{\prime-1 *}}{\longrightarrow}(q) g^{\prime}-1 * E\right]
$$

where $\left[\mathcal{F}_{t} \stackrel{q}{\rightarrow} E\right]$ is an arbitrary $T$-point of $\operatorname{Quot}_{\mathcal{F} / Z / S}^{P_{0}}$ sitting over $t \in S(T)$. That this action is algebraic will be clear when we embed $\mathbf{Q u o t}_{\mathcal{F} / Z / S}^{P_{0}}$ in a Grassmannian $S$-scheme via the Grothendieck embedding. This will be remarked shortly in the proof of the 2nd part. Let $p_{T}$ be the canonical morphism $\operatorname{Quot}_{\mathcal{F} / Z / S}^{P_{0}}(T) \rightarrow S(T)$. Then we have

$$
p_{T}\left(\left[\mathcal{F}_{t} \stackrel{q}{\rightarrow} E\right] \cdot g\right)=p_{T}\left[\mathcal{F}_{t \cdot g} \rightarrow\left(g^{\prime}-1\right)^{*} E\right]=t \cdot g=p_{T}\left(\left[\mathcal{F}_{t} \stackrel{q}{\rightarrow} E\right]\right) \cdot g,
$$

and it follows that $p_{T}$ is $\Gamma$-equivariant.

2. Existence of an $S$-very ample, $\Gamma$-linearized line bundle on $\operatorname{Quot}_{\mathcal{F} / Z / S}^{P_{0}}$ : Since $\mathcal{F}$ is $S$-flat, $f_{*} \mathcal{F}(n)=f_{*}\left(\mathcal{F} \otimes L_{1}^{n}\right)$ is locally free for all sufficiently large $n$. Choose such an $n$, and consider the Grothendieck embedding followed by the Plücker embedding of $S$-schemes (Proposition 3.8 of [9], Propostion 2.2.5 of [1]]):

$$
\operatorname{Quot}_{\mathcal{F} / Z / S}^{P_{0}} \stackrel{i_{G}}{\hookrightarrow} \operatorname{Grass}^{P_{0}(n)}\left(f_{*} \mathcal{F}(n)\right) \stackrel{i_{P}}{\hookrightarrow} \mathbb{P}\left(\left(\bigwedge^{P_{0}(n)} f_{*} \mathcal{F}(n)\right)^{*}\right) .
$$

Note that the linearization of $\mathcal{F}$ and that of $L_{1}$ give a $\Gamma$-action on the vector bundle $f_{*} \mathcal{F}(n)$, hence on the Grassmannian scheme and the projective bundle $P_{0}(n)$

$\mathbb{P}\left(\bigwedge f_{*} \mathcal{F}(n)^{*}\right)$. Note also that $i_{G}$ and $i_{P}$ are $\Gamma$-equivariant with respect to these $P_{0}(n)$

actions. (Therefore, $\operatorname{Quot}_{\mathcal{F} / Z / S}^{P_{0}}$ is a $\Gamma$-invariant subscheme of $\mathbb{P}\left(\bigwedge f_{*} \mathcal{F}(n)^{*}\right)$. It is clear that the $\Gamma$-action on this projective bundle is algebraic and compatible with the $\Gamma$-action on the base $S$. Thus it follows that the $\Gamma$-action on $\operatorname{Quot}_{\mathcal{F} / Z / S}^{P_{0}}$ defined in the proof of 1 is indeed algebraic and $p: \operatorname{Quot}_{\mathcal{F} / Z / S}^{P_{0}} \rightarrow S$ is $\Gamma$-equivariant.) Let $Q$ denote $\operatorname{Quot}_{\mathcal{F} / Z / S}^{P_{0}}$, and let $\mathcal{O}_{Q} \otimes_{\mathcal{O}_{S}} \mathcal{F} \rightarrow \mathcal{E} \rightarrow 0$ be the universal quotient over $Q \times{ }_{S} Z$. Then $\operatorname{det}\left(p_{Q *} \mathcal{E}(n)\right)=\operatorname{det}\left(p_{Q *}\left(\mathcal{E} \otimes L_{2}^{n}\right)\right)$ is the pull-back of $\mathcal{O}_{\mathbb{P}(\mathcal{L})}(1)$ (Proposition 3.8, [9], and Proposition 2.2.5, [11]), where $\mathcal{L}=\bigwedge^{P_{0}(n)} f_{*} \mathcal{F}(n)^{*}$. Hence it is very ample relative to $p$. But $\mathcal{O}_{\mathbb{P}(\mathcal{L})}(1)$ is the dual of the line bundle associated 
to the $k^{*}$-bundle $\mathcal{L} \rightarrow \mathbb{P}(\mathcal{L})$, which certainly has a $\Gamma$-action on it coming from the linearization of $\mathcal{F}$. Therefore, $\Gamma$ acts on $\mathcal{O}_{\mathbb{P}(\mathcal{L})}(1)$ by the dual action, and hence on $\operatorname{det}\left(p_{Q *} \mathcal{E}(n)\right)$, as $i_{G}$ and $i_{P}$ are $\Gamma$-equivariant.

The following very useful result is due to C. S. Seshadri [15].

Proposition 2. Let $\Gamma$ be a reductive algebraic group acting on projective algebraic schemes $S$ and $Z$, the actions being linear with respect to ample line bundles $L, M$ on $S, Z$ respectively. Let $p: Z \rightarrow S$ be a $\Gamma$-morphism. Suppose that $M$ is relatively ample with respect to $p$ and that $N$ is the line bundle $p^{*} L^{a} \otimes M^{b}(a, b \in \mathbb{Z}, a, b>0)$, which we write as $a L+b M$. Then if $b / a$ is sufficiently small (note that in this case $N$ is ample on $Z, c f . \S 4.6$, Chap. II [10]), we have the following:

1. $p^{-1}\left(S^{s}(L)\right) \subset Z^{s}(N)$,

2. $p\left(Z^{s s}(N)\right) \subset S^{s s}(L)$.

Remark 3. Though not quite relevant to the present article, it is worth remarking that this observation of Seshadri's accounts for a phenomenon familiar to the group of people working on augmented bundles. For instance, a Bradlow pair which consists of a vector bundle and a nonzero section of it has stabilities depending on a parameter usually called $\tau$. For special values of $\tau$, a Bradlow pair is $\tau$-stable if the bundle part is stable as a vector bundle. This is because the parameter comes from the linearization of Quot $\times \mathbb{P} V$, where Quot is a certain quot scheme parametrizing the bundle part and $\mathbb{P} V$ is the projective space parametrizing the section part, and those special values of $\tau$ are none other than the values that correspond to the linearizations with sufficiently small $b / a$ that we saw in the proposition above (with Quot $\times \mathbb{P} V$ as $Z$ and Quot as $S$.)

Combining Proposition 2 and Proposition [, we obtain the following corollary.

Corollary 1. Under the assumptions and notations of Proposition 1, we can choose a $\Gamma$-linearized ample line bundle $N$ on $\mathbf{Q u o t}_{\mathcal{F} / Z / S}^{P_{0}}$ such that the following holds:

1. $p^{-1}\left(S^{s}\left(L_{2}\right)\right) \subset\left(\operatorname{Quot}_{\mathcal{F} / Z / S}^{P_{0}}\right)^{s}(N)$.

2. $p\left(\left(\operatorname{Quot}_{\mathcal{F} / Z / S}^{P_{0}}\right)^{s s}(N)\right) \subset S^{s s}\left(L_{2}\right)$.

We also need the following simple lemma, which follows immediately from standard geometric invariant theory (for instance, p.6. of [12]).

Lemma 4. Let $\Gamma$ be an algebraic group over $k$, and $Z$ a scheme of finite type over $k$ with a $\Gamma$-action on it. Suppose that $Z$ has a geometric quotient $\phi: Z \rightarrow Z / / \Gamma$. Then any $\Gamma$-invariant open subscheme $Y$ of $Z$ has a geometric quotient.

Remark 4. The conclusion of the lemma is true for any immersion $i: Y \hookrightarrow Z$. One can easily prove the closed immersion case directly. (Or, it follows from the more general fact that if $i: Y \rightarrow Z$ is a finite morphism, then $Y$ has a geometric quotient [8].)

\section{Moduli of $k^{*} \times \operatorname{Aut}(\mathfrak{g})$-BUndLeS}

Let $G$ be a connected reductive algebraic group, and consider the immersive representation $k^{*} \times \operatorname{Aut}(\mathfrak{g}) \hookrightarrow \operatorname{GL}(\mathfrak{g})$. (Let $z \in k^{*} \cap \operatorname{Aut}(\mathfrak{g})$ in $\operatorname{GL}(\mathfrak{g})$. Then $[z x, z y]=z[x, y]$ implies $z=1$, and it follows that the representation is immersive.) Let $\mathcal{E} \rightarrow \mathcal{R} \times X$ be the $\mathrm{GL}(V)$-universal family of stable vector bundles with Hilbert polynomial $P$ as in the last section. By Lemma 3.(2), we obtain a GL( $V)$-universal 
family $\mathcal{E}_{1} \rightarrow \mathcal{R}_{1} \times X$ of $k^{*} \times \operatorname{Aut}(\mathfrak{g})$-bundles from $\mathcal{E} \rightarrow \mathcal{R} \times X$. We shall see in the next section that to construct a moduli scheme of stable $G$-bundles, it is enough to prove the existence of a geometric quotient $\mathcal{R}_{1} / / \mathrm{GL}(V)$. This quotient is, by Lemma 耳, a moduli scheme of $k^{*} \times \operatorname{Aut}(\mathfrak{g})$-bundles. The proof of the existence of such a quotient in 13 depends on the fact that $X$ is a nonsingular complete curve. (The considerably simpler construction in 2 also works for curves only.) We will accomplish it in this section over any projective scheme, using the results we obtained in the last section.

Consider the space $\mathfrak{g}^{*} \otimes \mathfrak{g}^{*} \otimes \mathfrak{g}=\operatorname{Hom}(\mathfrak{g} \otimes \mathfrak{g}, \mathfrak{g})$ of algebra structures of $\mathfrak{g}$, whose subvariety determined by the equation of Jacobi identity precisely consists of the Lie algebra structures of $\mathfrak{g}$. Let ad $\in \mathfrak{g}^{*} \otimes \mathfrak{g}^{*} \otimes \mathfrak{g}$ be the point defined by the given Lie algebra structure of $\mathfrak{g}$. With the obvious GL( $\mathfrak{g})$-action on $\mathfrak{g}^{*} \otimes \mathfrak{g}^{*} \otimes \mathfrak{g}$, the stabilizer of the line generated by ad is $k^{*} \times \operatorname{Aut}(\mathfrak{g})$, so that we have a $\operatorname{GL}(\mathfrak{g})$-equivariant embedding GL $(\mathfrak{g}) /\left(k^{*} \times \operatorname{Aut}(\mathfrak{g})\right) \hookrightarrow \mathbb{P}\left(\mathfrak{g}^{*} \otimes \mathfrak{g}^{*} \otimes \mathfrak{g}\right)$. This induces an embedding $\mathcal{E} /\left(k^{*} \times \operatorname{Aut}(\mathfrak{g})\right) \hookrightarrow \mathcal{E}\left(\mathbb{P}\left(\mathfrak{g}^{*} \otimes \mathfrak{g}^{*} \otimes \mathfrak{g}\right)\right)=\mathbb{P}\left(\mathcal{E}^{*} \otimes \mathcal{E}^{*} \otimes \mathcal{E}\right)$, and subsequently, an embedding $\operatorname{Hilb}_{\mathcal{R}}\left(\mathcal{E} /\left(k^{*} \times \operatorname{Aut}(\mathfrak{g})\right)\right) \hookrightarrow \operatorname{Hilb}_{\mathcal{R}}\left(\mathbb{P}\left(\mathcal{E}^{*} \otimes \mathcal{E}^{*} \otimes \mathcal{E}\right)\right)$. Then the GL(V)action on $\mathcal{E}$ carries over to $\mathbb{P}\left(\mathcal{E}^{*} \otimes \mathcal{E}^{*} \otimes \mathcal{E}\right)$ compatibly with the $\mathrm{GL}(V)$-action on $\mathcal{R}$, and consequently to $\mathbf{H i l b}_{\mathcal{R}}\left(\mathbb{P}\left(\mathcal{E}^{*} \otimes \mathcal{E}^{*} \otimes \mathcal{E}\right)\right)$, so that we have a $\mathrm{GL}(V)$-equivariant immersion $\mathcal{R}_{1} \hookrightarrow \mathbf{H i l b}_{\mathcal{R}}\left(\mathbb{P}\left(\mathcal{E}^{*} \otimes \mathcal{E}^{*} \otimes \mathcal{E}\right)\right.$ ). (Recall from Section 1 that $\mathcal{R}_{1}$ is an open subscheme of $\operatorname{Hilb}_{\mathcal{R}}(\mathcal{E} / H) \hookrightarrow \operatorname{Hilb}_{\mathcal{R}}\left(\mathbb{P}\left(\mathcal{E}^{*} \otimes \mathcal{E}^{*} \otimes \mathcal{E}\right)\right)$.)

Theorem 1. There exists a geometric quotient $\mathcal{R}_{1} / / \mathrm{GL}(V)$ of $\mathcal{R}_{1}$ by $\mathrm{GL}(V)$.

Proof. As remarked above, $\mathcal{R}_{1}$ is a $\mathrm{GL}(V)$-invariant subscheme of the Hilbert scheme $\mathbf{H i l b}_{\mathcal{R}}\left(\mathbb{P}\left(\mathcal{E}^{*} \otimes \mathcal{E}^{*} \otimes \mathcal{E}\right)\right)$. Thus by Lemma 4 and Remark 4 , it suffices to show that $\operatorname{Hilb}_{\mathcal{R}}\left(\mathbb{P}\left(\mathcal{E}^{*} \otimes \mathcal{E}^{*} \otimes \mathcal{E}\right)\right)$ has a geometric quotient. For this end, we first note that the composite morphism $\mathbb{P}\left(\mathcal{E}^{*} \otimes \mathcal{E}^{*} \otimes \mathcal{E}\right) \stackrel{\pi}{\rightarrow} \mathcal{R} \times X \rightarrow \mathcal{R}$ is flat. In fact, since $\pi$ is projective and $\pi_{*} \mathcal{O}_{\mathbb{P}\left(\mathcal{E}^{*} \otimes \mathcal{E}^{*} \otimes \mathcal{E}\right)}(n)=\operatorname{Sym}^{n}\left(\mathcal{E}^{*} \otimes \mathcal{E}^{*} \otimes \mathcal{E}\right)$ is locally free for all $n \geq 1$, we know that $\pi$ is flat. The projection $\mathcal{R} \times X \rightarrow \mathcal{R}$ is flat (being the base change of the flat morphism $X \rightarrow$ Spec $k)$, and therefore $\mathbb{P}\left(\mathcal{E}^{*} \otimes \mathcal{E}^{*} \otimes \mathcal{E}\right)$ is flat over $\mathcal{R}$. Hence we can apply Proposition 1 and Corollary 1 and conclude that there is a $\mathrm{GL}(V)$-linearized ample line bundle $N$ on $\mathbf{H i l b}_{\mathcal{R}}\left(\mathbb{P}\left(\mathcal{E}^{*} \otimes \mathcal{E}^{*} \otimes \mathcal{E}\right)\right)$ such that $p^{-1}\left(\mathcal{R}^{s}\right) \subset \mathbf{H i l b}_{\mathcal{R}}\left(\mathbb{P}\left(\mathcal{E}^{*} \otimes \mathcal{E}^{*} \otimes \mathcal{E}\right)\right)^{s}(N)$, where $p$ is the canonical projective morphism $\mathbf{H i l b}_{\mathcal{R}}\left(\mathbb{P}\left(\mathcal{E}^{*} \otimes \mathcal{E}^{*} \otimes \mathcal{E}\right)\right) \rightarrow \mathcal{R}$. But since $\mathcal{R}^{s}=\mathcal{R}$ by definition of $\mathcal{R}$, we conclude that

$$
\operatorname{Hilb}_{\mathcal{R}}\left(\mathbb{P}\left(\mathcal{E}^{*} \otimes \mathcal{E}^{*} \otimes \mathcal{E}\right)\right)^{s}(N)=\operatorname{Hilb}_{\mathcal{R}}\left(\mathbb{P}\left(\mathcal{E}^{*} \otimes \mathcal{E}^{*} \otimes \mathcal{E}\right)\right)
$$

and that $\operatorname{Hilb}_{\mathcal{R}}\left(\mathbb{P}\left(\mathcal{E}^{*} \otimes \mathcal{E}^{*} \otimes \mathcal{E}\right)\right)$ has a geometric quotient.

\section{Moduli of Stable $G$-Bundles}

In this section, we show how the existence of the quotient $\mathcal{R}_{1} / / \mathrm{GL}(V)$, proven in the last section, leads to the construction of the moduli scheme of $G$-bundles. The content of this section is shown in detail in 13. Since the adjoint representation $G \rightarrow \mathrm{GL}(\mathfrak{g})$ is not immersive, we need to break it into 4 steps as follows:

$$
G \longrightarrow G / Z \stackrel{\operatorname{Ad}}{\hookrightarrow} \operatorname{Aut}(\mathfrak{g}) \stackrel{1 \times 1_{\text {Aut }(\mathfrak{g})}}{\longrightarrow} k^{*} \times \operatorname{Aut}(\mathfrak{g}) \hookrightarrow \mathrm{GL}(\mathfrak{g}) .
$$

1. Let $\mathcal{E} \rightarrow \mathcal{R} \times X$ be the $\mathrm{GL}(V)$-universal family for $\mathrm{GL}(\mathfrak{g})$-bundles whose associated vector bundles are stable. Since $k^{*} \times \operatorname{Aut}(\mathfrak{g}) \rightarrow \mathrm{GL}(\mathfrak{g})$ is immersive, by Lemmas 2 and 3 we get a universal family for the set of $k^{*} \times \operatorname{Aut}(\mathfrak{g})$-bundles 
$E$ whose associated $\mathfrak{g}$-bundles $(E \times \mathfrak{g}) /\left(k^{*} \times \operatorname{Aut}(\mathfrak{g})\right)$ are stable. We denote the universal family thus obtained by $\mathcal{E}_{1} \rightarrow \mathcal{R}_{1} \times X$, as before.

2. A $k^{*} \times \operatorname{Aut}(\mathfrak{g})$-bundle $E$ comes from an Aut $(\mathfrak{g})$-bundle, or has reduction of structure to $\operatorname{Aut}(\mathfrak{g})$, if and only if the associated line bundle $E\left(k^{*}\right)=p_{1 *} E$ is trivial, where $p_{1}$ denotes the first projection $k^{*} \times \operatorname{Aut}(\mathfrak{g}) \rightarrow k^{*}=\left(k^{*} \times \operatorname{Aut}(\mathfrak{g})\right) / \operatorname{Aut}(\mathfrak{g})$. Now, the family of line bundles $\mathcal{E}_{1}\left(k^{*}\right) \rightarrow \mathcal{R}_{1} \times X$ corresponds to a morphism $f$ from $\mathcal{R}_{1}$ into the Picard scheme $\operatorname{Pic}(X / k)$. Let $\mathcal{R}_{1}^{\prime}=f^{-1}\left(\left[\mathcal{O}_{X}\right]\right)$, where $\left[\mathcal{O}_{X}\right]$ is the class of the trivial line bundle. It is the closed subscheme of $\mathcal{R}_{1}$ which parametrizes exactly those bundles whose associated $k^{*}$-bundles are trivial, or equivalently, those who have reduction of structure to $\operatorname{Aut}(\mathfrak{g})$. We conclude that

$$
\left.\mathcal{E}_{1}\right|_{\mathcal{R}_{1}^{\prime}} \rightarrow \mathcal{R}_{1}^{\prime} \times X
$$

is a $\mathrm{GL}(V)$-universal family for $\operatorname{Aut}(\mathfrak{g})$-bundles whose associated $\mathfrak{g}$-bundles are stable.

3. Let $\mathcal{E}_{1}^{\prime}=\left.\mathcal{E}_{1}\right|_{\mathcal{R}_{1}^{\prime}}$. Again by Lemma 2] $\Gamma\left(\mathrm{Ad}, \mathcal{E}_{1}^{\prime}\right)$ is representable and we get a $\operatorname{GL}(V)$-universal family $\mathcal{E}_{2} \rightarrow \mathcal{R}_{2} \times X$ for $\operatorname{Ad}(G)=G / Z$-bundles whose associated $\mathfrak{g}$-bundles are stable. Furthermore, $\mathcal{R}_{2} \rightarrow \mathcal{R}_{1}^{\prime}$ is étale and finite. This follows from the fact that the projection $\operatorname{GL}(\mathfrak{g}) / \operatorname{Ad}(G) \rightarrow \operatorname{GL}(\mathfrak{g}) / \operatorname{Aut}(\mathfrak{g})$ is an étale covering, combined with Lemma 4.14.1 of [13].

4. To construct a $\operatorname{GL}(V)$-universal space for stable $G$-bundles from $\mathcal{E}_{2} \rightarrow \mathcal{R}_{2} \times X$, we first construct a universal family for stable $G / G^{\prime} \times G / Z$-bundles, where $G^{\prime}=$ $(G, G)$ denotes the derived. Since $G$ is reductive, $G$ is the semidirect product $Z(G)^{\circ} \cdot G^{\prime}$ of the connected center and the derived, and hence $G / G^{\prime}$ is a torus $k^{* l}$ for some $l$. Then the $l$-fold product of the Picard scheme $\Pi^{l} \operatorname{Pic}(X / k)$ classifies $G / G^{\prime}$-bundles. Let $P \rightarrow \operatorname{Pic}(X / k)$ be a Poincaré bundle, and consider the following family of $G / G^{\prime} \times G / Z$-bundles:

$$
\mathcal{P} \times{ }_{X} \mathcal{E}_{2} \rightarrow\left(\Pi^{l} \operatorname{Pic}(X / k) \times \mathcal{R}_{2}\right) \times X,
$$

where $\mathcal{P}=P \times_{X} P \times_{X} \cdots \times_{X} P$ is the $l$-fold fibre product of $P$ over $X$. This is a $\operatorname{GL}(V)$-universal family of $G / G^{\prime} \times G / Z$-bundles whose associated $\mathfrak{g}$-bundles are stable. Let $\mathcal{E}_{2}^{\prime}=\mathcal{P} \times_{X} \mathcal{E}_{2}$. Then the associated sheaf $\Gamma\left(\rho, \mathcal{E}_{2}^{\prime}\right)^{\sim}$, where $\rho$ denotes the projection $G \rightarrow G / G^{\prime} \times G / Z$, is representable by the scheme $\mathcal{R}_{3}$ defined by the following Cartesian diagram (Lemma 4.15.1, 13]):

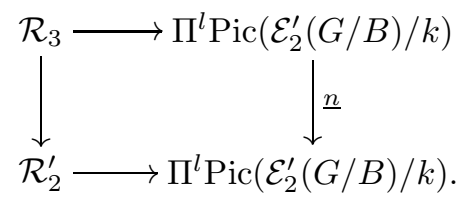

Here, $B$ is a suitable Borel subgroup and $\underline{n}$ is the morphism sending $\left(L_{1}, \ldots, L_{l}\right)$ to $\left(L_{1}^{\otimes n_{1}}, \ldots, L_{l}^{\otimes n_{l}}\right)$, for some $n_{1}, \ldots, n_{l} \in \mathbb{N}$. Since $\underline{n}$ is étale and finite, it follows that $\mathcal{R}_{3} \longrightarrow \mathcal{R}_{2}^{\prime}$ is also étale and finite.

5. Since $\mathcal{R}_{1}^{\prime} \longrightarrow \mathcal{R}_{1}$ is a closed immersion, it is finite. Also, we have remarked that $\mathcal{R}_{2} \longrightarrow \mathcal{R}_{1}^{\prime}$ is a finite étale morphism. Thus if $\mathcal{R}_{1}$ has a geometric quotient by $\mathrm{GL}(V), \mathcal{R}_{2}$ has a geometric quotient, by Lemma 5.1 of [13]. It is then clear that $\mathcal{R}_{2}^{\prime}=\Pi^{l} \operatorname{Pic}(X / k) \times_{X} \mathcal{R}_{2}$ has a geometric quotient, since $\mathrm{GL}(V)$ acts only on the second factor. Finally, since $\mathcal{R}_{3} \longrightarrow \mathcal{R}_{2}^{\prime}$ is finite, $\mathcal{R}_{3}$ has a geometric quotient which is by Lemma 1 the moduli scheme of stable $G$-bundles. Now, in the previous section, we have shown that $\mathcal{R}_{1}$ has a geometric quotient, and therefore we have proved the existence of a moduli scheme of stable $G$-bundles. 
Theorem 2. There exists a quasi-projective coarse moduli scheme $M_{G, P}$ for the functor $\underline{\mathbf{M}}_{G, P}$ (Definition 3) of stable principal G-bundles with Hilbert polynomial $P$.

\section{Stability in the Sense of Ramanathan}

In the last section, we compare our definition of stability with the following stability condition of Ramanathan.

Definition 6 (14]). Let $X$ be a nonsingular complex projective variety.

1. A principal $G$-bundle $E \rightarrow X$ is stable (resp. semistable) with respect to the polarisation $\mathcal{O}_{X}(1)$ if for any reduction of the structure group of $E$ to a maximal parabolic subgroup $P \subset G$ over any open subset $U \subset X$ with $\operatorname{codim}(X-U) \geq 2$, the line bundle associated to any character dominant with respect to a Borel subgroup contained in $P$ has degree $<0$ (resp. $\leq 0)$.

2. $E \rightarrow X$ is quasi-stable (or polystable) if it has a reduction $E_{M} \subset E$ of the structure group to a Levi component (i.e. a maximal reductive subgroup) $M$ of a parabolic subgroup $P \subset G$ such that the $M$-bundle $E_{M}$ is stable and for any character of $M$ trivial on the center of $G$ the line bundle associated to $E_{M}$ via the character has degree zero.

Then Ramanathan and Subramanian proved that a holomorphic $G$-bundle over a complex projective manifold $X$ is polystable if and only if it carries a (unique) Einstein-Hermitian connection. If $E$ is polystable in the sense of Ramanathan, then $E(\mathrm{GL}(\mathfrak{g}))$ is polystable by Theorem 3 of [14. Therefore, upon a suitable compactification which includes semistable bundles, our moduli space would contain all the polystable bundles in the sense of Ramanathan. The author thanks Professor V. Balaji for pointing this out and drawing his attention to [14.

\section{ACKNOWLEDGMENT}

The author is greatly indebted to Professor William Haboush, who suggested this problem and guided him to the solution presented here. He also wishes to thank Professor Steven Bradlow for many discussions, and encouragement. Grateful thanks are due also to those who read an early version and gave helpful comments and encouragement, especially to Professor V. Balaji and Professor Ch. Sorger. Lastly, the author is very grateful to the referee for a careful review and many comments which made this article much better.

\section{REFERENCES}

[1] P. Aspinwall, and R. Donagi, The Heterotic String, the Tangent Bundle, and Derived Categories, Adv.Theor.Math.Phys. 2 (1998) 1041-1074. MR 2000b:81116

[2] B. Balaji and C.S, Seshadri, Semistable principal bundles, Preprint (1999).

[3] R. Donagi, Taniguchi Lecture on Principal Bundles on Elliptic Fibrations, Integrable Systems and Algebraic Geometry (Kobe/Kyoto, 1997; M.-H. Saito et al., eds.), World Sci. Publ., River Edge, NJ, 1998, pp. 33-46. MR 2000a:14015

[4] _ Principal bundles on elliptic fibrations, Asian J. Math. 1 (1997), 214-223. MR 99d:14010

[5] R. Friedman and J. W. Morgan, Holomorphic principal bundles over elliptic curves, Preprint (1998), math.AG/9811130.

[6] R. Friedman and J. W. Morgan, Holomorphic principal bundles over elliptic curves II : The parabolic construction, Preprint (2000), math.AG/0006174. 
[7] R. Friedman, J. W. Morgan, and E. Witten, Principal G-bundles over elliptic curves, Math. Res. Lett. 5 (1998), 97-118. MR 99j:14037

[8] D. Gieseker, On the moduli of vector bundles on an algebraic surface, Ann. of Math. 106 (1977), 45-60. MR 81h:14014

[9] A. Grothendieck, Technique de decente et théorèmes d'existence en géométrie algébrique IV. Les Schémas de Hilbert., Séminaire Bourbaki, t. 13, 1960/61, no. 221. MR 27:1339

[10] A. Grothendieck et J. Dieudonné, Éléments de Géometrie Algébrique, Inst. des Hautes Études Sci., Publ. Math. Nos. 4, 8, 11, 17, 22, 24, 28, 32 (1960-67). MR 29:1210, MR 30:3885, MR 33:7330 MR 36:177. MR 36:178 MR 39:220

[11] D. Huybrechts and M. Lehn, The Geometry of Moduli Spaces of Sheaves, Aspects of Mathematics, E 31, Vieweg (1997). MR 98g:14012

[12] D. Mumford, J. Fogarty, F. Kirwan, Geometric Invariant Theory, Third Enlarged Edition, Springer-Verlag (1994). MR 95m:14012

[13] A. Ramanathan, Moduli for principal bundles over algebraic curves I, II, Proc. Indian Acad. Sci. (Math. Sci.), 106 (1996), 301-328 and 421-444. MR 98b:14009

[14] A. Ramanathan and S. Subramanian, Einstein-Hermitian connections on principal bundles and stability, J. reine. angew. Math. 390 (1988), 21-31. MR 90b:32057

[15] C. S. Seshadri, Quotient spaces modulo reductive algebraic groups, Ann. Math., 95 (1972) 511-556. MR 46:9044

[16] C. Simpson, Moduli of representations of the fundamental group of a smooth projective variety I, II, Inst Hautes Études Sci. Publ. Math. 79 (1994), 47-129 and 80 (1995), 5-79. MR 96e:14012 MR 96e:14013

Department of Mathematics, University of Illinois, 1409 W. Green Street, Urbana, ILLINOIS 61801

Current address: Department of Mathematics, Rice University, 6100 S. Main St., Houston, Texas 77005

E-mail address: hyeon@math.rice.edu 